\title{
Verba peregrina: Von der Interdiktion zur Integration
}

\author{
Roman Müller
}

\begin{abstract}
Summary
Varro in De lingua latina was the first to draw a distinction between authentic Latin words (verba nostra) and lexical elements of foreign origin (verba peregrina). The aim of this paper is to examine the different attitudes of Roman authors and grammarians towards foreign elements in general. Specific attention is paid to the various arguments against lexical borrowing (lack of comprehensibility, offence to Latinitas, comption of style by barbarisms, etc.) as well as to the role of grecisms in Latin linguistic thought. Among the factors that have brought about a gradual change from interdiction to integration, five are discussed more closely: (1) the poetic licence (sermo poetarum): (2) the growing need for technical and special terms; (3) the general authorization to use words from every part of Italy (Quintilian); (4) the increasing association of lasinitas with consuetudo; (5) the revolution of linguistic theory caused by Christian authors. Finally, the paper shows how grammarians in the late Latin period turned away from banishing foreign words and preferred only to set up rules of fitting them into the traditional language system.
\end{abstract}

Das Thema der verba peregrina ergibt sich aus einer der beliebten Zweiteilungen, mit denen Varro in De lingua latina die Vielfalt des Sprachlichen zu ordnen und zu beschreiben suchte. Soweit unsere Kenntnis der römischen Sprachreflexion reicht, erscheint er als der erste, der die Wörter des Lateinischen aufgrund der etymologischen Ausgangsfragen cur sint, unde sint? systematisch in zwei Klassen gliederte, in die eigenen und die fremden. Die eigenen sind bei ihm die verba oder vocabula nostra, vernacula, Latina; die fremden die verba oder vocabula peregrina, auch adventicia, aliena'. Wenngleich bei späteren Autoren die Terminologie variierte, blieb die Zweiteilung des Wortschatzes als lexikalisches Prinzip aufrechterhalten. In Bewunderung Varros hat Quintilian sie ausdrücklich mit dem Lehrsatz bekräftigt:

(1) uerba aut Latina aut peregrina sunt (Quint. inst. 1,5,55).

\footnotetext{
' ling. 5,3: neque omnis origo est nostrae linguae e vernaculis verbis; 5,10: verba. quae sumt aut nostra aut aliena aut oblivia; 5,77: aquatilium vocabula animalium partim sunt vernacula, partim peregrina; 5,100: Ferarum vocabula item partim peregrina; 5,103sq.: Quae in hortis nascuntur, alia peregrinis vocabulis, ut Graecis... Vernacula [sc. verba]...; 5,167: In his multa peregrina...; 10,70: adventicia pleraque habemus Graeca; 10,69: Drei regelmäBige Deklinationstypen (genera) gibt es, umum vernaculum ac domi natum, alterum adventicium, tertium nothum ex peregrino hic natum.
} 
Mit feinerer Untergliederung, vor allem der Unterscheidung von Lehnwort und Fremdwort im engeren Sinn, behauptet sich die Trennung des Wortschatzes in einen angestammten Teil und einen aus anderen Sprachen hinzugekommenen auch noch in der modernen Sprachwissenschaft.

Varro stützte sein Zweierschema auf die pythagoreische Lehre, wonach allem Seienden das Prinzip binärer Gegensätze zugnundeliege. Doch betrifft dieser Rekurs nur die gedankliche Ordnung seines Wortmaterials. Die grundsätzliche Erkenntnis, daß die Wörter sprachgeschichtlich nicht nur eigenen, sondern auch fremden Quellen entstammen, reicht von ihm über seinen Lehrer Aelius Stilo weit in die griechische Sprachreflexion zurück. Es fehlt hier die Zeit, die Problementwicklung im Griechischen darzulegen. Deshalb beginne ich gleich mit einer notwendigen Begriffsklärung.

Was ist ein verbum peregrinum in dem Moment, da diese Bezeichnung in den ersten Belegen entgegentritt? Befragt man die Etymologie, so wird auf das Adverb peregre zurückverwiesen, eine Lokalbestimmung zu allem, quae extra patriae terminos sita sunt, sive illius agro adiacent sive mari separantur ${ }^{2}$. Etymologisch betrachtet, ist das verbum peregrinum also ein Sprachelement von jenseits des ager - d. h. des Gebiets - lateinsprechender Bevölkerung. Die Kennzeichnung bringt von Hause aus einen strikten Gegensatz von Latein und Nicht-Latein zum Ausdruck. Wie im hellenozentrischen Denken der Griechen die Welt in die der eigenen Sprache und in die der Barbaren zerfiel, so erschien in dieser Konzeption der Erdkreis grundsätzlich zweigeteilt zwischen der Sprache Roms und dem ganzen anderen, dem 'fremden' Sprachraum. Doch diese Auffassung muBte bald modifiziert werden; die Beweggründe zeichnen sich schon in Varros Umgang mit dem Begriff der verba peregrina ab.

Wenn Varro nämlich von verba peregrina (oder auch verba adventicia, verba aliena) redet, so umgeht er im Lateinischen absichtsvoll die Charakterisierung verba barbara. Sie hätte bei der Übernahme des dualistischen Sprachenmodells der Griechen am nächsten gelegen. Varro kennt sie auch, doch reserviert er barbarus in seinen Schriften bezeichnenderweise für die Unterscheidung vocabula nostra : vocabula barbara durch den Pergamener Krates von Mallos, d. h. er hält sie frei für die Beschreibung der Sprachensituation aus griechischer Sicht ${ }^{3}$. Hieran geht auf, daß wegen der Hochachtung der römischen Eliten für alles Griechische es sich für ihn verbot, Entlehnungen aus der Nachbarsprache im selben Atemzug wie die aus lateinfernen Idiomen einfach 'barbarisch' zu nennen. Während Varro also mit verba peregrina der Spracheneinteilung aus römischer Perspektive gerecht zu werden versuchte, erzwang das Problem, wie man der herausgehobenen Rolle des Griechischen und der Minderbewertung der sonstigen Entlehnungen besser entsprechen könne, auf Dauer doch eine begriffliche Korrektur.

\footnotetext{
${ }^{2}$ ThLL X, 1, 10 sqq.

${ }^{3}$ Varro ling 8,64.
} 
Als zweiter kritischer Punkt kündigt sich bei Varro die ungleiche Bewertung der Spendersprachen Italiens an. Nur Entlehnungen aus dem Gallischen und dem Lukanischen erscheinen bei ihm ausdrücklich als verba peregrina ${ }^{4}$. Dutzende von Wörtern aus den italischen Schwestersprachen Sabinisch, Oskisch, Umbrisch, Faliskisch, und selbst Anleihen beim Etruskischen und beim sizilianischen Griechisch werden dagegen so ausgewiesen, wie wenn es sich um lateinnahes Sprachgut handelte. Eine eigentümliche Affinität des Lateinischen aus alter Zeit meint Varro zum Äolischen und sogar zum archaischen Griechisch ausmachen zu können. Spielt bei der Beurteilung der inneritalienischen Beziehungen das Vorbild der griechischen Dialektvielfalt die ausschlaggebende Rolle, so ist die Verbindung zum Äolischen und zum frühen Griechischen mit dem Euander-Mythos zu erklären, wonach der Heros des arkadischen Pallantion durch seine Niederlassung auf dem Boden des späteren Rom die Verschmelzung des Äolisch-Griechischen mit dem Lateinischen begründet habe. Verstärkt durch den Aeneas-Mythos und die Sage von der Ansiedlung einiger Gefährten des Herakles in Latium, hat die These von der hereditären Gräzität des Lateinischen mit umso größerer Dringlichkeit die Entscheidung der Frage verlangt, ob Entlehnungen aus dem Griechischen gleichermaßen als verba peregrina anzusprechen seien wie die aus dem Gallischen, Hispanischen, Armenischen, Syrischen oder Phönikischen, die Varro mit entsprechender Kennzeichnung zitiert. Wie dieses Problem behandelt wurde, ist zunächst zu betrachten.

Eindeutige Dokumente einer begrifflichen Absonderung der nichtgriechischen Entlehnungen von den griechischen haben wir erst aus nachklassischer Zeit: Quintilian bezeugt die Trennung in der Institutio Oratoria 1,5,7sqq., wo er bei der Klassifizierung der barbarismi eine besondere Art unterscheidet, die darin bestehe, daß “jemand der lateinischen Rede ein afrikanisches oder hispanisches Wort einfügt":

(2) barbarismum pluribus modis accipimus. unum gente, quale sit si quis Afrum uel Hispanum Latinae orationi nomen inserat (Quint. inst. 1,5,7 sq.).

Quintilian dehnt die Beispiele jedoch auch auf Wörter aus dem "Gallischen" und dem "Sardischen" aus. Das heiBt: Abweichend von den bisherigen Definitionen rechnet er die Aufnahme beliebiger fremder Wörter nichtgriechischer Herkunft zu den vitia des Barbarismus, während Anleihen beim Griechischen nicht darunter fallen. Von Plinius d. Ä. an mehren sich die Zeugnisse, die innerhalb der 'fremden' Wörter die 'barbarischen' ausgliedern. Im 4. Jh. schließlich bilden Grammatiker für den Neologismus nichtgriechischer Herkunft über die Kollokation barbaros lexis eigens die Bezeichnung barbarolexis aus ${ }^{5}$.

\footnotetext{
4 ling. 5,167 führt er als vocabula peregrina an das "gallische" sagum (mit richtiger Zuordnung) und das "gallische" reno (das aber aus dem Germanischen stammt). Zu den vocabula peregrina der Tiernamen zählt er ling. 5, 100 mit möglicher "lukanischer Herkunft" das (lat. oder illyr.) Wort ursus. "Die maBgebende Begriffsbestimmung stammt von Donatus: Eine lexikalische Regelwidrigkeit in nostra loquella barbarismus, in peregrina barbarolexis dicitur, ut siquis dicat mastruga cateia magalia (Keil GL IV, 392, 5sq.). Von Servius erhalten wir die Bestätigung, daB auch andere zu seiner
} 
Die offenkundige Neigung, zwischen griechischen und nichtgriechischen Elementen qualitativ und terminologisch eine deutliche Trennlinie zu ziehen, blieb nicht ohne Rückwirkung auf die Verwendungsbreite des Allgemeinbegriffs der verba peregrina. Bei Autoren, die Wert darauf legten, verba barbara von den griechischen Wörtern zu sondern, finden wir ihn daraufhin überwiegend eingeschränkt auf Gräzismen. Immerhin hält aber noch ein Grammatiker wie der Afrikaner Pompeius im 5./6. Jh. an der Überordnung der verba peregrina über die barbarolexis fest ${ }^{6}$, wie er es bei seinem Vorbild Donatus gelesen hat. Auch andere Autoritäten bezeugen, daB die Bezeichnung verba peregrina neben dem inzwischen vorherrschenden engeren Bezug auf Gräzismen bisweilen noch immer den generellen auf alles Nichtlateinische bewahren konnte.

Warum hat sich gegen fremde Wörter im Lateinischen Kritik erhoben? Varros erhaltene Schriften, die als erste die Unterscheidung zwischen verba nostra und verba peregrina treffen, bekunden, von einem Seitenhieb auf die Gräkomanie abgesehen, nur ein überwiegend philologisches Interesse an der Differenzierung. Doch hatte sich in der vorklassischen Rhetorik eine Doktrin entfaltet, nach der die Unterscheidung sinnvoll war. Ich meine die Anweisung an den Redner, Unverständlichkeit und Unklarheit zu vermeiden. Die Rhetorica ad Herennium hatte bereits eingeschärft, daB die Gewähltheit des Ausdrucks durch Reinheit und Klarheit erreicht werde. Die Klarheit hänge davon $a b$, daB man gebräuchliche und zutreffende Wörter verwende, wobei als gebräuchlich diejenigen zu gelten hatten, die in der Umgangssprache vorkamen ${ }^{7}$. In besonderem $\mathrm{MaB}$ war Klarheit für das exordium und dann für die narratio der Rede gefordert, denn der Sachverhalt einer causa lieB sich nur mit einem vertrauten, sofort verständlichen Vokabular darstellen. Ausdrücklich warnte die Herennius-Rhetorik nicht nur vor veralteten Wörtern, sondern auch vor verba nova, weil deren Sinn dem Publikum weitgehend verborgen bliebe. Wurden die verba peregrina in diesem Zusammenhang auch nicht eigens erwähnt, dürfen wir sie, sofern sie nicht schon seit langem ins Lateinische integriert waren, zu den zu vermeidenden Problem- und Neuwörtem rechnen, die sich in einer Rede aus Gründen der Verständlichkeit verboten.

$\mathrm{Da}$ alle folgenden Rhetoriken das Klarheitsprinzip mit ähnlicher Entschiedenheit verfochten, war daraus die unbeugsame Ablehnung ungewohnter und erst recht neu übernommener Entlehnungen abzuleiten.

Zeit den Ausdruck zur Kennzeichnung nichtgriechischer verba peregrina gebraucht haben: $s i$ peregrina sint verba (habemus enim multa et a Gallis et ab Africis et ab aliis gentibus), tumc barbarolexis vocanur (Keil, GL IV, 444, 7sq.). Die Ausbreitung des Begriffs hat Raija Vainio (1999) kompetent untersucht (bes. 83-96).

${ }^{6}$ Keil $G L$ V, 284, 19-21.

${ }^{7}$ Rhet. Her. 4,17: Explanatio est, quae reddit apertam et dilucidam orationem. Ea conparatur duabus rebus, usitatis verbis et propriis. Usitata sunt ea, quae versantur in /sermone] consuetudine cotridiana. 
Mit dem Gebot der Klarverständlichkeit ging von der Herennius-Rhetorik an das Postulat sprachlicher Reinheit einher. Hieraus ergab sich zum pragmatischen Impuls zusätzlich ein normativer, auf die Wortwahl zu achten. Der Appell, nur mit puris et electis verbis vor die Öffentlichkeit zu treten (Rhet. Her. 4,16), verengte sich gegen Ende der Republik zur Weisung, den stadtrömischen sermo urbanus zum MaBstab des guten und richtigen Lateins, der latinitas, zu nehmen, also alles von außen Hereindrängende fermzuhalten. Wenn Cicero, der Protagonist der stadtrömischen Norm, von verba quaedam non trita Romae sprach (Cic. Brut. 171), so wamte er davor, daB lexikalischer Zustrom aus Gallien und anderswoher das Ideal des sermo purus beflecken könnte. Doch sah er die Gefahr nicht nur in den Wanderwörtern aus eroberten Ländern, sondem auch in einer Überfremdung Roms mit den zugewanderten oder als Sklaven herbeigeschafften Allophonen, welche das Risiko mit sich brachten, daß sie das korrekte Latein mit dem weiterwirkenden Substrat ihrer fremden Sprachen durchsetzten. In urbem nostram est infusa peregrinitas, schreibt Cicero in der epist. ad fam. 9,15 und beklagt, daB schon keine Spur der alten Feinheit mehr da sei. Quintilian folgte der Linie seines großen Vorbilds mit der Mahnung, die Reden müBten so wenig fremd und ausländisch wie möglich sein, in allen Wörtern und in der Artikulation den Sohn Roms verraten, aber nicht so, als sei ihnen das Bürgerrecht nur verliehen worden:

(3) hic non alienum est admonere ut sint quam minime peregrina et externa (...) quare, si fieri potest, et verba omnia et vox huius alumnum urbis oleant, ut oratio Romana plane videatur, non civitate donata. (Quint. inst. 8,1,2-3).

Wie man sieht, insistierte er im Hinblick auf den unterschiedlichen Status von cives und peregrini im Gemeinwesen auf einem sozusagen bürgerrechtlichen Abstand zwischen angestammten, d.h. vollgültigen, und fremden, also minderberechtigten sprachlichen Elementen. Cicero hatte sich da noch weit unnachsichtiger gegeben. Im Brutus hatte er die Klage über den schlechten EinfluB der allophonen Zuwanderer auf das Lateinische mit dem ersten Aufruf zur Sprachreinigung verknüpft, den die Sprachgeschichte kennt:

(4) confluxerumt enim et Athenas et in hanc urbem multi inquinate loquentes ex diversis locis. quo magis expurgandus est sermo (Cic. Brut. 258).

Ein solch scharfer Ton war in der vorausgegangenen griechischen Sprachreinheitsdiskussion nie angeschlagen worden; er hat sich aber auch in Rom nicht zu einer allgemeinen Sprachpolitik gesteigert.

Cicero markiert bezeichnenderweise auch den Wendepunkt in der Selbsteinschätzung der Römer gegenüber der griechischen Sprache und Kultur, einen Wendepunkt, der das Verhältnis zu den Gräzismen beeinflussen sollte. Wir wissen freilich schon von älteren $\mathrm{Au}$ toren, daB sie im Bestehen auf dem Eigenwert des Lateinischen Front gemacht hatten gegen die maßlose Bewunderung und Kopie des Griechischen bei ihren Zeitgenossen. Ein sehr frühes, vielsagendes Zeugnis liefert hier Terenz. Wie sich in einer lexikalischen Studie seiner Komödien nachweisen ließ, hat dieser Dramatiker im Gegensatz zum geradezu grä- 
komanen Plautus höchstens acht Neologismen vom Griechischen ins Lateinische geholt; alle übrigen Gräzismen seiner Stücke waren schon vor ihm aufgenommen und assimiliert worden ${ }^{8}$. Von daher gesehen gewinnt das Lob des Iulius Caesar für Terenz, er sei puri sermonis amator', eine neue Bedeutung. Auch für Varro schien es einen Unterschied auszumachen, ob ein Wort griechischer Herkunft zum Altbestand des Lateinischen zählte, gewissermaßen zur ursprachlich gemeinsamen lingua prisca, oder aber zu den Entlehnungen jüngeren Datums. Ein Hinweis in dieser Richtung läßt sich einer Stelle in De re rustica entnehmen, wo Varro sich mokant darüber aufhält, daB inzwischen jeder Gutshof und jede Gegend einen Fischteich brauche, eine piscina, die die philograeci jedoch amphibium nennen, und ein Gänsezuchtgehege, das bei ihnen chenoboscion heiße ${ }^{10}$.

Ohne die Faszination und die kulturtragende Rolle des Griechischen im geringsten herabzusetzen, hat Cicero sein Sprachprogramm darauf ausgerichtet, der verbreiteten These von der patrii sermonis egestas mit dem Ausbau des Lateinischen zu einem nicht nur ebenbürtigen, sondern überlegenen Medium des geistigen Austausches entgegenzutreten. Interessant ist dabei, wie er mit dem Problem der Gräzismen verfuhr, die ja die Superiorität des Griechischen zu bestätigen schienen. Charakteristischerweise suchte er Gräzismen in den Reden zu vermeiden. Von denen, die er verwandte, waren die meisten längst eingebürgert gewesen. In den philosophischen und rhetorischen Schriften fällt die Zahl der Anleihen wegen der unvermeidbaren Fachtermini naturgemäB höher aus ", doch begegnet dort auch immer wieder Ciceros Ausweichpraktik, das fremde Wort in griechischer Form zu bringen, um so das Lateinische freizuhalten, oder aber die Übernahme durch eine Lehnübersetzung oder Periphrase zu vermeiden. Fabricemus, si opus erit, verba rät er in Academica 1,17 für den Fall eines wirklichen lexikalischen Neubedarfs. Nach dem Wortlaut in De fin. 3,5 zu schließen, hat Cicero neben einer vorhandenen lateinischen Bezeichnung prinzipiell nur dann eine von griechischer Herkunft dulden wollen, wenn diese schon lange gebräuchlich gewesen war ${ }^{12}$. Der Billigung des alteingewurzelten Lehnguts bei

${ }^{8}$ Dazu Roman Müller 1996: 254-265. Über die maximal acht von Terenz selbst eingeführten Gräzismen informiert S. 262. J. Blänsdorf kommt im Artikel 'Terentius' (DNP 12/1, 2002, 151) auf das Ergebnis mit der Bemerkung zurück, "von den 93 griech. Wörtern waren fast alle bereits eingebürgert".

9 Überliefert in der Vita Terenti Suetons, die Donatus am Anfang seines Terenz-Kommentars wiedergibt (ed. Wessner 1902-05: 1-10).

${ }^{10}$ Varro rust. 3,10,1: Transi, inquit Axius, nunc in illud genus. quod non est [ulla] villa ac terra contentum, sed requirit piscinas, quod vos philograeci vocatis amphibium. in quibus ubi anseres aluntur, nomine aliquo chenoboscion appellatis.

"Vgl. M. v. Albrecht 1973: $1242 \mathrm{f}$.

12 Cic. de fin. 3,5: ea verba, quibus instituto verernm utimur pro Latinis, ut ipsa philosophia, ut rhetorica, dialectica, grammatica, geometria, musica, quamquam Latine ea dici poterant, tamen, quoniam usu percepta sum, nostra ducamus. 
gleichzeitiger Absage an alle Neologismen, folglich auch an solche aus dem Griechischen, schloB sich im 1. und 2. Jh. n. Chr. die archaisierende Richtung der Literatur an.

Sowohl in ihrer Gesamtheit wie auch in ihrer problematischen Aufteilung in längst heimisch gewordene und 'neue' Entlehnungen hatten die Gräzismen natürlich von vornherein einen Schwachpunkt in der Abwehr fremden Wortguts gebildet, aber sie allein haben den Widerstand gegen Neologismen von draußen nicht ins Wanken bringen können, betrachtete doch eine Bildungselite in Rom das Griechische gar nicht als etwas dem Lateinischen Fernstehendes, sondern als Zweitidiom, ging daher auch von "unseren beiden Sprachen" aus: utroque sermone nostro, wie Sueton formulierte ${ }^{13}$. Was hat dann letztlich in der Einstellung gegenüber den verba peregrina einen Wandel in Gang gebracht? Es kamen in der Entwicklung des Lateinischen mehrere Umstände zusammen, die zunächst einzelne Teilgebiete der Sprachverwendung von der Ablehnung ausnahmen, aber insgesamt am Ende dazu führten, daB das Veto nur noch als verbale Pflichtübung artikuliert worden ist.

In dieser Hinsicht konnte nicht ohne Folgen bleiben, daß Grammatiker und Rhetoriker von vornherein darauf verzichtet hatten, ihren Regelungen auch die Dichtersprache zu unterwerfen. Die Anerkennung der Dichtersprache als sermo poetarum, als eines Sprachbereichs eigener Gesetzlichkeit neben dem sermo oratorum und dem sermo cottidianus, hatte sich bereits in der Rhetorica ad Herennium abgezeichnet ${ }^{14}$. Varro baute sie aus zur Doktrin, der poeta habe alle Freiheiten der Sprachgestaltung. Seine Wörter und Formen dürten sich abheben von denen des Volkes, auch von denen des Redners, denn sie gehorchten nicht demselben Recht: quod eorum non idem ius. Das Volk habe sich nach der RegelgemäBheit zu richten, der Redner nicht immer, während der Dichter "alle Grenzen straflos überschreiten dürfe" ${ }^{15}$. Ihre Rechtfertigung bezog die poetische Lizenz für Varro aus dem speziellen und altertümlichen Vokabular der Dichtung, das nicht nur die verba prisca des Lateinischen zu bewahren schien, sondern auch die Seherkraft der veteres poetae. Spätere Sprachautoritäten begründeten die Freiheit lieber mit den Erfordernissen des Wohlklangs und der Metrik in der Poesie. Was nun die Lizenz in Bezug auf den Fremdwortgebrauch angeht, so ist von Bedeutung, daB derselbe Quintilian, der die Verwendung eines nichtgriechischen verbum peregrinum zu den Erscheinungsformen des barbarismus zählte, in einer Erläuterung nachtrug, daß jeder VerstoB, den der Grammatiklehrer einem Dichter ankreiden könnte, dagegen Nachsicht oder sogar Lob verdiene:

(5) Sed quidam fere in iactationem eruditionis sumere illa ex poetis solent, et auctores quos praelegunt criminantur. Scire autem debet puer haec apud scriptores carminum aut uenia digna aut etiam laude duci... (Quint. inst. 1,5,11).

${ }^{1.3}$ Suet. Claud. 42.

${ }^{14}$ Die Rhet. Her. 4,43 unterscheidet klar zwischen den drei Bereichen der consuetudo poetarum, der consuetudo oratorum und des cottidianus serno. Sprachmuster der poetae und der oratores vorzustellen, gehört ohnehin zur Lehrpraxis $(2,34,25 ; 2,34,36 ; 4,1,15 ; 4,2,18)$.

${ }^{15}$ Varro ling. 9,5 . 
Was in der Rhetorik und im allgemeinen Sprachgebrauch als vitium zu brandmarken war, konnte sich also in der Poesie zur virtus wandeln. Von daher rührt das Anliegen spätlateinischer Sprach- und Dichtungslehren, dem Begriff des barbarismus in der poetischen Funktion den des lizenzgedeckten metaplasmus gegenüberzustellen ${ }^{16}$. Welch großes Ermessen die dichterische Freiheit in der Wortwahl gewährte, führt z.B. das Aufgebot nichtlateinischer Vokabeln in Vergils Werken vor Augen.

Ebenso wenig wie im Bereich der Poesie griff das Verdikt der Grammatiker und Rhetoriker im fachsprachlichen Sektor des Lateinischen. Hier weitete sich das Einsickern der verba peregrina in demselben Tempo zum Strom, wie das Fachschriftum immer weitere Gebiete des Wissens erschloB. Signifikant ist hier ein Verhalten Catos des Älteren. Damit das Lateinische nicht in seiner angestammten Substanz gefährdet werde, riet er in den Libri ad Marcum filium seinem Sohn dringend, sich aus griechischen Büchem zwar das überlegene Wissen der Nachbarn anzueignen, doch diese Bücher nicht bis zum Auswendiglemen zu studieren. Dem Hüter altrömischer Tugend und Sprache kam es nur darauf an, daB der Kenntnisstand der Griechen für Rom nutzbar gemacht wurde; sprachlich sollte es keine Beeinflussung geben ". Umso bezeichnender war es für den fachterminologischen Ausbaubedarf des Lateinischen aber, daB derselbe Cato nicht umhin konnte, in sein Werk über die Landwirtschaft De agri cultura eine Vielzahl neuer Gräzismen aufzunehmen, alles fachspezifische Bedarfsentlehnungen. Als der Sprachpurismus von der klassischen Ära ab auch das Fachschriftum zu erfassen drohte, umgingen die Autoren die lexikalischen Einengungen, indem sie die fremden Termini mit metalinguistischen Formeln zitierten, die sie hinnehmbar machten, z. B. ut dicunt Graeci; graece A, vulgo B appellatur; quod graece $A$; etc. ${ }^{18}$. Die ErschlieBung der Sach-, Tier- und Pflanzenwelt ferner Länder sowie die Eingliederung immer weiterer Gebiete in das Imperium überspülten das Lateinische schlieblich mit Exotismen und Regionalbezeichnungen ohne Schranken, nach dem ciceronischen Gesetz:

(6) renum copia verborum copiam gignit. (Cic. de orat. 3,125,2).

Eine Vorstellung davon erhält man bereits bei der Lektüre der Namen der Wasser- und Wildtiere sowie der Pflanzen, die Varro in De lingua larina bespricht ${ }^{19}$.

\footnotetext{
${ }^{16}$ Zum metaplasmus H. Lausberg 1960: $\$ \$ 479-495$. Die Entwicklung des Begriffs in Abgrenzung zu dem des barbarismus hat Raija Vainio in der Studie 'Barbarism and metaplasm' grundlegend geklärt (Vainio 1999: 123-148).

${ }^{17}$ Auf das gespaltene Verhältnis Catos zum Griechischen und auf die hier angesprochene Thematik geht auch Rudolf Weis in Müller/Werner/Sier 1992: 137-142, ein.

${ }^{18}$ Die Zitiertechniken, mit deren Hilfe griechische Fachtermini verwendbar gemacht wurden, hat $O$. Wenskus 1996 erschöpfend dargestellt. Dort auch Behandlung des Bildes von der Verleihung des römischen Bürgerrechts an Wörter aus anderen Sprachen. Wichtig zur Problematik fremder Termini außerdem Wenskus 1998.

${ }^{19}$ ling. 5,77sq.; 5, 100sq.; 5,103.
} 
Der Hispanier Quintilian hat offenbar erkannt, daB auch die Beschränkung des lexikalischen Standards auf den sermo urbanus der stadtrömischen Oberschicht der Ausdruckskapazität des kanonisierten Lateins in einem idiomatisch, kulturell und real doch vielfältig gegliederten Großraum sowie in einer auf Geschichtswerke, Fachbücher und Enzyklopädien ausgeweiteten Literatur allzu enge Grenzen zog. Nur so läBt sich erklären, daB er sich in puncto Wortschatz in einem entscheidenden Schritt von der starren Romfixiertheit seines Vorbilds Cicero löste und Italien aus der Sperrklausel ausnahm. "Fremde Wörter (verba peregrina) sind, wie ich sagen möchte, ebenso wie die Menschen und wie auch viele Gewohnheiten von fast allen Völkem zu uns gekommen", leitet er seine Stellungnahme zur Entlehnung ein, um am Ende zu befinden:

(7) licet omnia Italica pro Romanis habeam. (Quint. inst. 1,5,55sq.)

"Alles aus Italien Stammende möchte ich für römisch halten".

Legt man dieser Aussage den damaligen geographisch-politischen Begriff von Italia zugrunde, so umfaBte die Erweiterung den ganzen Sprachraum von Sizilien bis zu den Alpen. Das heißt: Quintilian stellte Regionalismen aus dem griechischen Sizilien, dem etruskischen Gebiet, der gallischen Po-Ebene, dem illyrischen Küstensaum an der Adria, dem rätischen Alpenraum usw. dem Wortschatz Roms gleichberechtigt zur Seite.

War es einerseits der Zwang der Verhältnisse, der den Widerstand gegen die verba peregrina abbaute, kamen andererseits im Spätlatein auch sprachtheoretische und christliche Motive der Tolerierung hinzu. Noch einmal ist Quintilian als Wegbereiter hervorzuheben. Bei ihm nämlich erhielt der Sprachgebrauch, die consuetudo, den Rang des obersten Sprachrichtigkeitskriteriums, wodurch die Rückbindung der latinitas an die traditionellen Muster der auctoritas und der vetustas, also an die Vergangenheit, ins Wanken geriet ${ }^{20}$. Es war ferner von Bedeutung, daB die Christen eine grundlegend neue Bewertung der Sprachen aufbrachten. An Stelle des alten Gegensatzes zwischen dem Griechischen bzw. Lateinischen und den 'barbarischen' Idiomen propagierten sie das Gegenüber von christlicher und heidnischer Sprachwelt, das alle Völker und Zungen des neuen Glaubens in eine Gemeinschaft verwandelte. Barbarisch sei nur die Sprache, die Gott nicht preisen kann, machte Augustinus klar ${ }^{21}$. Die Kirchenväter sahen auch keine Veranlassung zu rechtfertigen, daB die christliche Lehre mit Gräzismen, Hebraismen und sonstigen Neologismen in großer Zahl einherkam, sich außerdem um das Verbot von Barbarismen und Soloezismen des Lateinischen nicht scherte; denn derartige Formalien waren, wie unter anderen Arnobius argumentierte ${ }^{22}$, völlig bedeutungslos für die inhaltliche Tragweite und das Verstehen

\footnotetext{
${ }^{20}$ Zur consuetudo als Sprachrichtigkeitskriterium Roman Müller 2001: 183-207. Den Aufstieg zur wichtigsten Entscheidungsinstanz untersucht im besonderen Roman Müller 2003.

${ }^{21}$ Zur Sprachentrennung zwischen der una lingua in fide cordis aller Völker der Gläubigen und den Idiomen der dem Heil noch fernstehenden gentiles A. Borst 1958: 395ff.

an adv. gentes $1,58 \mathrm{sq}$.
} 
der Botschaft. Ein vorherrschend pragmatisches Interesse an der Sprache hob in der christlichen Lehre die Allgemeinverbindlichkeit der Gesetze des sermo purus et Latinus auf.

Kennzeichnend für den Auffassungswandel ist das Desinteresse der Sprachlehrer des 5. und 6. Jhs., die scharfe Unterscheidung des fremden, nichtgriechischen Wortes vom eigenen aufrechtzuerhalten und auf das aktuelle Vokabular anzuwenden. Während Grammatiker und Kommentatoren des 4. Jhs. z. B. die barbarolexis rigoros verdammten, ist bei den Späteren zu beobachten, daB sie den Terminus zum Teil wieder fallen ließen und die Kritik an der Wortentlehnung zu pflichtschuldigen Bemerkungen mit der Erwähnung weniger stereotyper Beispiele abschwächten, meistens der schon von Donatus zitierten. An Stelle von Ausführungen zur Wortwahl nehmen nach dem 4. Jh. Fragen des praktischen Umgangs mit den verba peregrina den gröBten Raum ein: Wie läBt sich der fremde Wortakzent mit dem lateinischen vereinbaren ${ }^{23}$ ? Wie fügt sich die fremde Form in das eigene Genussystem $^{24}$ ? Und vor allem: Wie kommt man in der Flexion mit der Latinisierung hin, wenn man möglichst einer Regel folgt, wie sie Consentius festgehalten hat:

omne peregrinum nomen, siquidem iam receptum est..., dirigi ad eius latini sermonis similitudinem debet, cui proximum est (Keil, GL V,364,8sqq).

Es geht, wie man sieht, nach dem 4. Jh. in der Sprachlehre gar nicht mehr um das Problem, ob der Wortschatz um verba peregrina erweitert werden darf, auch nicht um die Akzeptabilităt der einen oder anderen Übernahme, sondern nur noch um die systemverträgliche Integration der von irgendwoher bezogenen Neuheiten - dies freilich um einen hohen Preis. Denn abgesehen davon, daß abgestimmte umfassende Regelungen nicht mehr erreicht werden konnten, zog die Öffnung des Vokabulars auch dessen regionale Differenzierung nach sich, die Ausbildung spezifischer lexikalischer Sprachräume, die zur Ausformung romanischer Dialekte und damit zum Verlust der Einheit beigetragen hat.

\section{Bibliographie}

v. Albrecht, Michael (1973), M. Tullius Cicero, "Sprache und Stil", in: RE Suppl. 13, 1237-1347.

Borst, Arno (1958), Der Turmbau von Babel, II, 1, Stuttgart.

Lausberg, H. (1960), Handbuch der literarischen Rherorik, I, München.

Müller, Roman (2003), "Rhetorik und sprachlicher Paradigmenwechsel in der lateinischen Spätantike", in: H. Solin \& M. Leiwo \& H. Halla-aho (Hrsg.), Latin Vulgaire - Latin tardif VI, Actes du VF colloque international sur le latin vulgaire et tardif, Hildesheim, 53-61.

Müller, Roman (2001), Sprachbewußtsein und Sprachvariation im lateinischen Schrifttum der Antike, München (Zetemata, 111).

${ }^{23}$ Die Akzentuierung wird schließlich freigestellt, so Diomedes (Keil GL 1, 31sqq.), Cledonius (V, 33, 18sq.), Priscian (III, 520, 23sq.).

${ }^{24}$ Die Genusdiskussion konzentriert sich auf peregrine Wörter mit auslautendem -i (wie gummi, sinapi). Die Entscheidung bleibt zwischen Neutrum und Femininum in der Schwebe, vgl. Consentius (Keil $G L$ V, 347, 25), Pompeius (IV, 165, 8sq.), Priscian (II, 205, 1sq.). 
Müller, Roman (1996), Sprechen und Sprache: Dialoglinguistische Studien zu Terenz, Heidelberg. Vainio. Raija (1999), Latinitas and Barbarisms according to the Roman Grammarians, Turku.

Weis. Rudolf (1992), "Zur Kenntnis des Griechischen im Rom der republikanischen Zeit", in: C. W. Müller \& K. Sier \& J. Werner (Hrsg.), Zum Umgang mit fremden Sprachen in der griechischrömischen Antike [...]. Stuttgart.

Wenskus, Otta (1996), "Markieren der Basissprache in lateinischen Texten mit griechischen Einschaltungen und Entlehnungen", in: IF 101, 233-257.

Wenskus, Otta (1998), "Reflexionen zu fachsprachlichen Phänomenen in der Antike und Spätantike", in: L. Hoffmann \& H. Kalverkämper \& H. E. Wiegand (Hrsg.), Fachsprache [...], 1, 1, Berlin New York, $295-301$.

Wessner, Paulus (1902-05) (Hrsg.), Donatus, Aelius, Commentvm Terenti, Lipsiae. 
